Staphylococcus aureus (MRSA) or Pseudomonas aeruginosa. Mice randomly received a resorbable antibody delivery vehicle at the implant site: a blank carboxymethylcellulose (CMC) aqueous gel or the same CMC gel containing $10 \mathrm{mg}$ of pooled polyclonal human immunoglobulin G locally on the implant after infection, either alone or in combination with systemic doses of cefazolin or vancomycin antibiotics. Human antibodies were rapidly released (first-order kinetics) from the gel carrier to both peritoneal fluids and serum in both infection scenarios. Inocula required for lethal infection were substantially reduced by surgery and the presence of the implant versus a closed lethal peritonitis model. Survival to 10 days with two different $P$. aeruginosa strains was significantly enhanced $(P<.01)$ by the direct application of CMC gel containing antibodies alone to the surgical implant site.

Human-equivalent doses of systemic vancomycin provided a significantly improved benefit $(P<.01)$ against lethal, implant-centered, gram-positive MRSA infection. However, locally delivered polyclonal human antibodies in combination with a range of systemic vancomycin doses against MRSA failed to improve host survival. Successful antibody therapy against gram-negative, implant-centered infections complements the clinically routine use of systemic antibiotics, providing a mechanism of protection independent of antibiotic resistance.

FROM: Poelstra KA, Barekzi NA, Rediske AM, Felts AG, Slunt JB, Grainger DW. Prophylactic treatment of gram-positive and gram-negative abdominal implant infections using locally delivered polyclonal antibodies. $J$ Biomed Mater Res 2002;60:206-215.

\section{Fibrin Sheath Enhances Central Venous Catheter Infection}

Mehall and colleagues from the University of Arkansas for Medical Sciences, Little Rock, Arkansas, conducted a study to determine whether fibrin-coated central venous catheters have a higher infection rate, and spawn more septic emboli, than uncoated catheters after exposure to bacteremia. The study compared catheter infection and blood cultures of fibrin-coated and uncoated catheters exposed to bacteremia using adult male Sprague-Dawley rats.

A total of 210 rats had catheters placed with the proximal end buried subcutaneously. Rats were divided into three groups: tail vein bacterial injection on day 0 (no fibrin group) or on day 10 (fibrin group), or no injection/saline injection (control, $n=40$ ). Bacterial injections were $1 \times 10^{8}$ colony-forming units of either Staphylococcus epidermidis $(\mathrm{n}=100)$ or Enterobacter cloacae $(\mathrm{n}=60)$. Animals were killed 3 days after injection. Blood cultures were obtained via cardiac puncture, and catheters were removed via the chest. Half of the catheter was rolled onto agar and the other half was placed in trypticase soy broth. Plates and broth were incubated at $37^{\circ} \mathrm{C}$ for 48 hours. The presence of more than 15 colonies on roll plates, or growth in broth, was accepted as a positive sign of infection. Thirty animals without catheters had bacterial injections and had blood cultures 3 days after injection.

Catheter infection with S. epidermidis occurred in $32 \%$ of roll plates and $80 \%$ of broth from the fibrin group versus $4 \%$ of roll plates and $20 \%$ of broth from the no fibrin group $(P<.01$ for each). Catheter infection with $E$. cloacae occurred in $50 \%$ of roll plates and $80 \%$ of broth from the fibrin group versus $0 \%$ of roll plates and $12 \%$ of broth from the no fibrin group $(P<.01$ for each). Positive blood cultures occurred in 47 of 68 animals from the fibrin group versus 8 of 68 from the no fibrin group $(P<.01)$. Microscopy showed a fibrin sheath on 20 of 20 catheters. Without catheters, 30 of 30 blood cultures were negative.

The authors concluded that the fibrin sheath significantly enhanced catheter-related infection and persistent bacteremia.

FROM: Mehall JR, Saltzman DA, Jackson RJ, Smith SD. Fibrin sheath enhances central venous catheter infection. Crit Care Med 2002;30:908-912.

\section{P. aeruginosa Cells Adapted to Benzalkonium Chloride Are Not Antibiotic Resistant}

Loughlin and colleagues from the United Kingdom conducted studies to determine whether strains of Pseudomonas aeruginosa can adapt to growth in increasing concentrations of the disinfectant benzalkonium chloride, and whether co-resistance to clinically relevant antimicrobial agents occurs. Attempts were made to determine what phenotypic alterations accompanied resistance and whether these explained the mechanism of resistance. Strains were serially passaged in increasing concentrations of benzalkonium chloride in static nutrient broth cultures. Serotyping and genotyping were used to determine the purity of the cultures. Two strains were examined for crossresistance to other disinfectants and antibiotics by broth dilution minimum inhibitory concentration determination. Alterations in outer membrane proteins and lipopolysaccharide expressed were determined, as well as cell surface hydrophobicity and charge, uptake of disinfectant, and proportion of specific fatty acid content of outer and cytoplasmic membranes.

Two $P$. aeruginosa strains showed a stable increase in resistance to benzalkonium chloride. Co-resistance to other quaternary ammonium compounds was observed in both strains; chloramphenicol and polymyxin $\mathrm{B}$ resistance was observed in one and a reduction in resistance to tobramycin was observed in the other. However, no increased resistance to other biocides (chlorhexidine, triclosan, and thymol) or antibiotics (ceftazidime, imipenem, ciprofloxacin, and tobramycin) was detected. Characteristics accompanying resistance included alterations in outer membrane proteins, uptake of benzalkonium chloride, cell surface charge and hydrophobicity, and fatty acid content of the cytoplasmic membrane, although no evidence was found for alterations in lipopolysaccharide. Each of the two strains had different alterations in pheno- 